\title{
Genetic Association and Expression Correlation between Colony-Stimulating Factor 1 Gene Encoding M-CSF and Adult-Onset Still's Disease
}

\author{
Yi-Ming Chen $\mathbb{D}^{1},{ }^{1,2,3}$ Wei-Ting Hung, ${ }^{1,4}$ Wan-Chun Chang, ${ }^{5}$ Chia-Wei Hsieh, ${ }^{1,3}$ \\ Wen-Hung Chung, ${ }^{5,6,7,8}$ Joung-Liang Lan, ${ }^{9,10,11}$ Ning-Rong Gung $\mathbb{D}^{,}{ }^{9}$ Yun-Shien Lee, ${ }^{12}$ \\ Der-Yuan Chen $\mathbb{D}^{\mathbb{9}},{ }^{9,10,13}$ and Shuen-Iu Hung $\mathbb{D}^{5,6,7,8,14}$
}

${ }^{1}$ Division of Allergy, Immunology and Rheumatology, Department of Medical Research, Taichung Veterans General Hospital, 407 Taichung, Taiwan

${ }^{2}$ Faculty of Medicine, National Yang Ming University, 112 Taipei, Taiwan

${ }^{3}$ Ph.D. Program in Translational Medicine, National Chung Hsing University, 402 Taichung, Taiwan

${ }^{4}$ Institute of Clinical Medicine, National Yang Ming University, 112 Taipei, Taiwan

${ }^{5}$ Department of Dermatology, Drug Hypersensitivity Clinical and Research Center, Linkou Chang Gung Hospital,

333 Taoyuan, Taiwan

${ }^{6}$ School of Medicine, Chang Gung University, 333 Taoyuan, Taiwan

${ }^{7}$ Cancer Vaccine and Immune Cell Therapy Core Laboratory, Department of Medical Research, Chang Gung

Immunology Consortium, Chang Gung Memorial Hospital, Linkou, 333 Taoyuan, Taiwan

${ }^{8}$ Whole-Genome Research Core Laboratory of Human Diseases, Chang Gung Memorial Hospital, 204 Keelung, Taiwan

${ }^{9}$ Rheumatology and Immunology Center, China Medical University Hospital, 404 Taichung, Taiwan

${ }^{10}$ School of Medicine, China Medical University, 404 Taichung, Taiwan

${ }^{11}$ Rheumatic Diseases Research Laboratory, Rheumatology and Immunology Center, China Medical University Hospital, 404 Taichung, Taiwan

${ }^{12}$ Department of Biotechnology, Ming Chuan University, 333 Taoyuan, Taiwan

${ }^{13}$ Translational Medicine Laboratory, Rheumatology and Immunology Center, China Medical University Hospital, 404 Taichung, Taiwan

${ }^{14}$ Institute of Pharmacology, National Yang-Ming University, 112 Taipei, Taiwan

Correspondence should be addressed to Der-Yuan Chen; dychen1957@gmail.com and Shuen-Iu Hung; hungshueniu@gmail.com

Received 9 December 2019; Accepted 21 January 2020; Published 14 February 2020

Academic Editor: Francesca Santilli

Copyright (C) 2020 Yi-Ming Chen et al. This is an open access article distributed under the Creative Commons Attribution License, which permits unrestricted use, distribution, and reproduction in any medium, provided the original work is properly cited.

Adult-onset Still's disease (AOSD) is a rare and inflammatory disorder characterized by spiking fever, rash, arthritis, and multisystemic involvement. HLA has been shown to be associated with AOSD; however, it could not explain the innate immunity and autoinflammatory characteristics of AOSD. To assess the genetic susceptibility of AOSD, we conducted a genome-wide association study (GWAS) on a cohort of 70 AOSD cases and 688 controls following a replication study of 36 cases and 200 controls and meta-analysis. The plasma concentrations of associated gene product were determined. The GWAS, replication, and combined sample analysis confirmed that SNP rs11102024 on $5^{\top}$-upstream of CSF1 encoding macrophage colony-stimulating factor (M-CSF) was associated with AOSD $\left(P=1.20 \times 10^{-8}\right.$, OR (95\% CI): $\left.3.28(2.25 \sim 4.79)\right)$. Plasma levels of M-CSF increased in AOSD patients $(n=82$, median: $9.31 \mathrm{pg} / \mathrm{mL})$, particularly in the cases with activity score $\geq 6(n=42$, $10.94 \mathrm{pg} / \mathrm{mL})$, compared to the healthy donors $(n=68,5.31 \mathrm{pg} / \mathrm{mL})(P<0.0001)$. Patients carrying rs11102024TT genotype had higher M-CSF levels (median: $20.28 \mathrm{pg} / \mathrm{mL})$ than those with AA genotype $(6.82 \mathrm{pg} / \mathrm{mL})(P<0.0001)$ or AT genotype $(11.61 \mathrm{pg} / \mathrm{mL})(P=0.027)$. Patients with systemic pattern outcome were associated with elevated M-CSF and frequently observed in TT carriers. Our data suggest that genetic variants near CSF1 are associated with AOSD and the rs11102024 T allele 
links to higher M-CSF levels and systemic outcome. These results provide a promising initiative for the early intervention and therapeutic target of AOSD. Further investigation is needed to have better understandings and the clinical implementation of genetic variants nearby CSF1 in AOSD.

\section{Introduction}

Adult-onset Still's disease (AOSD) is an inflammatory disorder characterized by spiking fever, macular rash, leukocytosis, arthritis, variable multisystemic involvement, and increase of acute phase reactants $[1,2]$. It is a rare disease with crude prevalence of only $1-2$ cases in 100,000-1 million annually [3,4]. The clinical features and disease progression of AOSD vary considerably. In severe cases, AOSD may lend to permanent joint destruction, organomegaly, lymphadenopathy, serositis, and aseptic meningitis [5-7]. Due to its characteristics and predominate dysregulation of innate immunity, AOSD has been considered an autoinflammatory disease $[8,9]$. Standard treatments for AOSD include corticosteroids as the first-line, the use of nonsteroidal anti-inflammatory drugs (NSAIDs), and disease-modifying antirheumatic drugs (DMARDS) to manage the clinical symptoms $[6,10,11]$. Nevertheless, AOSD still lacks effective therapeutics, as its etiology and pathophysiology remain unclear [12].

The proposed pathomechanisms of AOSD involve the dysregulation of the immune system $[13,14]$, interaction between host and environment factors [15-17], and genetic susceptibility [18-21]. Important laboratory characteristics of AOSD are leukocytosis with predominance of neutrophils and the increase of proinflammatory cytokines, including IL- $1 \beta$, IL-6, IL-18, TNF- $\alpha$, and IFN $\gamma$, but with negative testing for rheumatoid factor (RF) and autoantibodies $[14,22,23]$. In particular, macrophage activation is a clinic feature of AOSD $[8,24]$. High levels of macrophage colonystimulating factor (M-CSF), a critical growth factor for macrophage differentiation and activation, have been observed in the plasma of AOSD patients [22, 25, 26]. The heterogeneous phenotypes and biosignature of AOSD imply the potential involvement of complex genetic predisposition.

Most of the previous genetic studies on AOSD revealed association with variants on human leukocyte antigen (HLA) class I and II regions, such as HLA-BW35, HLA$D R B 1 * 15$ and $H L A-D R B 1 * 04[19,20]$. However, the association was inconsistent and controversial among the various studies of different populations $[27,28]$. In addition, the results of genetic studies did not link to the pathogenesis, and none of them have been associated with AOSD disease outcomes [14, 28]. Here, we enrolled 106 patients and 888 population controls and applied GWAS discovery and subsequent replication analysis to investigate the genetic susceptibility of AOSD. We explored the correlation between the identified genetic risk factor(s) and disease severity or outcomes and examined the functional implication of the associated genetic variants in AOSD.

\section{Materials and Methods}

2.1. Patient and Public Involvement. This study was carried out following the rules of the Declaration of Helsinki of
1975, which was revised in 2013. This study was approved by the ethics committee of the Institutional Review Board (IRB) of Taichung Veterans General Hospital (CF13321), and the written consent was obtained from each participant. We enrolled a total of 106 AOSD patients fulfilling the Yamaguchi criteria [29] between January 2010 and December 2015. Patients with infections, malignancies, or other rheumatic diseases were excluded. The disease activity of AOSD was assessed with a modified Pouchot score described by Rau et al. [23]. According to the proposed classification of disease courses of AOSD [9], the AOSD patients with follow-up at least one year were classified into two patterns of disease outcomes: (i) the "systemic pattern" that includes the monocyclic and the polycyclic form and (ii) the "chronic articular pattern" (persistent arthritis involving at least one joint destruction and lasting longer than 6 months) [30]. All of the AOSD patients were unrelated Han Chinese. Seventy of them were randomly selected as the case group in the GWAS discovery cohort.

We recruited 924 ethnically and geographically matched healthy subjects as the population controls from a biobank under a nationwide population study, which comprises 9,980 Han Chinese descendants [31]. There was no selfreport of rheumatic diseases among the recruited controls from Taiwan, where $98 \%$ of the population is made up of Han Chinese. Of the 924 population controls, we randomly obtained 724 controls for the GWAS discovery cohort and the rest of 200 individuals were for the replication cohort, which was an independent analysis conducted by $36 \mathrm{AOSD}$ cases versus 200 population controls to validate the statistically significant SNPs derived from the initial GWAS results.

2.2. Genotyping and Quality Controls in the Genome-Wide Scan. Genomic DNA was extracted from the peripheral blood of the enrolled subjects using Flexi Gene DNA kits (Qiagen, Hilden, Germany). We performed GWAS on the samples obtained from 70 AOSD cases and 724 population controls using the Affymetrix SNPs Array 6.0 platform (Santa Clara, CA, USA), which is composed of 909,622 SNPs [31]. Briefly, $200 \mathrm{ng}$ of genomic DNA of each sample was PCR amplified, fragmented, precipitated, and resuspended in the appropriate hybridization buffer. After hybridization, the BeadChip oligonucleotides were extended by a singlelabeled base, which were detected by fluorescence imaging with an Affymetrix Bead Array Reader. Normalized bead intensity data obtained for each sample were loaded into the Affymetrix SNP Array 6.0 software, which converts the fluorescence intensities into SNP genotypes. The genotype calls were generated using the Birdseed method (Birdseed v2) with Affymetrix Power Tools (version, apt-1.10.2).

We analyzed the GWAS data by the software Plink (v1.90b5) using logistic regression modeling with covariates: sex, and ancestry-specific principal components (i.e., PC1, 
PC2, PC3, PC4, PC5, PC6, PC7, PC8, PC9, and PC10). The genomic coordinates are based on NCBI Human Genome Build 37 (GRCh37). Of the 909,622 genotyped SNPs, 868,494 SNPs located on the autosomal chromosome were included for the quality control (QC) process. Our quality control for SNPs was a call rate higher than 0.95 , and 59,494 variants were removed due to missing genotype data. We excluded 163,017 variants due to minor allele threshold(s) (minor allele frequency $(\mathrm{MAF})<0.01$ ). The quantile-quantile plot was generated using the R/Bioconductor package: GWASTools [32]. The genomic inflation factor was calculated by Plink (v1.90b5). Linkage disequilibrium (LD) analysis of the SNP rs11102024 was performed by using the LDproxy module of the online software package LDLink (https://analysistools.nci.nih.gov/LDlink).

2.3. Replication Analysis and Targeted Gene Sequencing. For the replication study, we applied TaqMan assays (Thermo Fisher Scientific, CA, USA) or direct sequencing on the associated SNPs revealed by the initial GWAS using an independent sample set of 36 AOSD cases and 200 population controls. The oligonucleotide primers for polymerase chain reaction (PCR) amplification of the SNP rs11102024 near CSF1 gene are forward primer: $5^{\prime}$-TCCTATTGCATTGG GCATATT- $3^{\prime}$, and reverse primer: $5^{\prime}$-TCCATTTACGCC TCAACTCA-3', and those for SNP rs9636107 near TCF4 gene are forward primer: $5^{\prime}$-GCTGGTACCAAGGAAAG CTG- $3^{\prime}$, and reverse primer: $5^{\prime}$-CCTGCTGGTGTTTTGT TTTG- $3^{\prime}$. The PCR reaction was performed in three steps: $3 \mathrm{~min}$ at $95^{\circ} \mathrm{C}$; then 40 cycles of 20 seconds at $95^{\circ} \mathrm{C}, 30 \mathrm{sec}-$ onds at $58^{\circ} \mathrm{C}$, and 30 seconds at $72^{\circ} \mathrm{C}$, followed by 7 minutes at $72^{\circ} \mathrm{C}$.

\subsection{Determination of Plasma Levels of Macrophage Colony-} Stimulating Factor (M-CSF). The plasma samples of 82 patients with AOSD were collected at the active status of the disease, and the patients received corticosteroids and/or the nonsteroidal anti-inflammatory drugs (NSAIDs). Besides, the disease-modifying antirheumatic drugs (DMARDs) had also been prescribed for the patients, which included methotrexate (patients number $(n)=74$ ), hydroxychloroquine $(n=66)$, cyclosporine $(n=30)$, sulfasalazine $(n=18)$, and azathioprine $(n=10)$. Levels of plasma M-CSF were determined on the samples from these 82 active AOSD patients and 68 population controls using enzyme-linked immunosorbent assay (ELISA) according to the manufacturer's instructions (Ray-Biotech Inc., GA, USA).

2.5. Statistical Analysis. For the GWAS, we conducted the statistical analysis for the associations by comparing the allele frequencies between AOSD cases and population controls. The SNP association was examined by Fisher's exact tests and rank-ordered according to the lowest $P$ value. All the $P$ values were two-tailed. The corrected $\mathrm{Pc}$ values were adjusted using Bonferroni's correction for multiple comparisons (645,983 for GWAS SNPs), and Pc value for genomewide significance should be $<7.8 \times 10^{-8}(0.05 / 645,983)$. The odds ratios $(\mathrm{OR})$ were calculated using Haldane's modification [33]. Levels of plasma M-CSF in the different groups
TABLE 1: Demographic data, clinical characteristics, and laboratory findings of 106 patients with adult-onset Still's disease (AOSD).

\begin{tabular}{lc}
\hline Characteristics $^{*}$ & AOSD patients \\
\hline Age at study entry (mean \pm SD, years) & $42.9 \pm 15.0$ \\
Females, $n(\%)$ & $72(67.9 \%)$ \\
Fever $\left(\geqq 39^{\circ} \mathrm{C}\right), n(\%)$ & $102(96.2 \%)$ \\
Evanescent rash, $n(\%)$ & $94(88.7 \%)$ \\
Arthralgia or arthritis, $n(\%)$ & $78(73.6 \%)$ \\
Sore throat, $n(\%)$ & $74(69.8 \%)$ \\
Liver dysfunction, $n(\%)$ & $42(39.6 \%)$ \\
Lymphadenopathy, $n(\%)$ & $39(36.8 \%)$ \\
Hepatosplenomegaly, $n(\%)$ & $14(13.2 \%)$ \\
White blood cell count & $16205 \pm 11673$ \\
(mean \pm SD, cells/microliter) & $351.5 \pm 151.0$ \\
Platelet count (mean $\left.\pm \mathrm{SD}, \times 10^{3} / \mathrm{cumm}\right)$ & $78.4 \pm 36.6$ \\
ESR values (mean $\pm \mathrm{SD}, \mathrm{mm} / 1^{\text {st }}$ hour) & $10.2 \pm 7.8$ \\
CRP levels (mean $\pm \mathrm{SD}, \mathrm{mg} / \mathrm{dL})$ & $8989 \pm 15328$ \\
Ferritin levels (mean $\pm \mathrm{SD}, \mu \mathrm{g} / \mathrm{L})$ & $5.48 \pm 1.11$ \\
Clinical activity score $(\mathrm{mean} \pm \mathrm{SD})$ &
\end{tabular}

${ }^{*}$ Data are presented as the mean \pm standard deviation (SD) or number (percentage). Liver dysfunction was defined as alanine aminotransferase (ALT) level $\geqq 40$ IU/L. ESR: erythrocyte sedimentation rate; CRP: C-reactive protein.

were compared by the nonparametric Mann-Whitney $U$ test. The differences in the frequencies of significant alleles among AOSD patients with different courses of disease outcomes were examined using Fisher's exact test. The correlation coefficient was obtained by nonparametric Spearman's rank correlation test. A probability of less than 0.05 was considered to be significant.

\section{Results}

3.1. Clinical Characteristics of AOSD Patients. The demographic data, clinical characteristics, and laboratory findings of 106 patients with AOSD are shown in Table 1. The mean age of the patients is 42.9 years (standard deviation (SD): 15.0 ), and the proportion of females is $67.9 \%$. The presence of spiking fevers $\left(\geq 39^{\circ} \mathrm{C}\right)$ is observed in $102(96.2 \%)$ cases, evanescent rash in $94(88.7 \%)$ cases, arthralgia or arthritis in $78(73.6 \%)$ cases, sore throat in $74(69.8 \%)$ cases, liver dysfunction in $42(39.6 \%)$ cases, lymphadenopathy in 39 $(36.8 \%)$ cases, and hepatosplenomegaly shown by sonography in $14(13.2 \%)$ cases, respectively. The mean of white blood cell count in the 102 patients is observed as 16,205 cells/microliter (SD: 11673), platelet count as $351.5 \times 10$ [3]/cumm (SD: 151.0), ESR (erythrocyte sedimentation rate) value as $78.4 \mathrm{~mm} / 1$ st hour (SD: 36.6), CRP (C-reactive protein) as $10.2 \mathrm{mg} / \mathrm{dL}$ (SD: 7.8), ferritin level as $8989 \mu \mathrm{g} / \mathrm{L}$ (SD: 15328), and clinical activity score as 5.48 (SD: 1.11).

3.2. Genome-Wide Scan of AOSD. We first conducted a GWAS using samples of 70 AOSD patients and 724 controls. After the QC process, 36 controls were removed, and 645,983 variants and 758 samples (including 70 cases and 688 


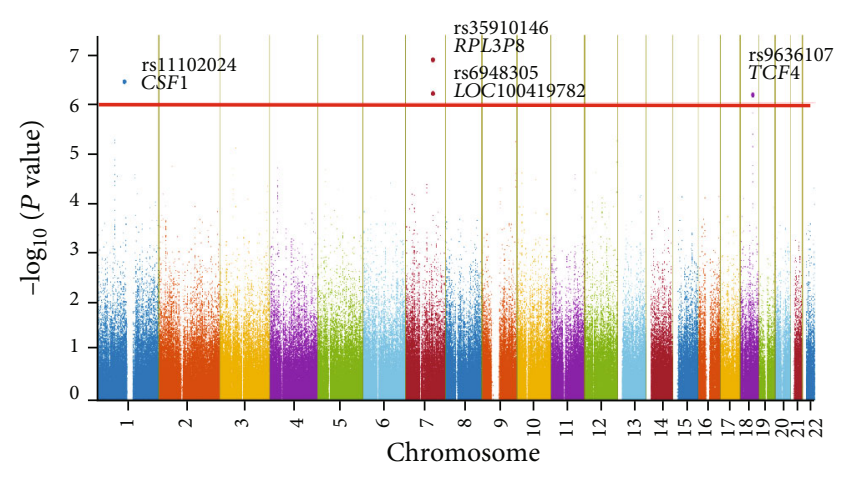

FIGURE 1: Genome-wide association scan for AOSD. Manhattan plot shows association between 645,983 SNPs and AOSD. Each dot represents a $-\log _{10}(P$ value $)$ derived with a logistic regression model adjusted by sex and principal components (PCs) in 70 AOSD cases and 688 population controls. The red horizontal line marks the level of significance suggestive of association $\left(P<1 \times 10^{-6}\right)$. The top 4 AOSD-associated variants are labeled with the name of the nearest gene(s). CSF1: colony-stimulating factor 1; RPL3P8: ribosomal protein L3 pseudogene 8; LOC100419782: pseudogene; TCF4: transcription factor 4.

population controls) passed the filters. The mean call rate is 98.3\%. The SNPs on sex chromosomes were excluded from the GWAS analysis. The principal component analysis (PCA) map showed that there is no difference in the distribution of ancestry among AOSD cases and population controls (Supplemental Figure 1). A quantile-quantile plot of the test statistics was used for quality control and revealed that the population matching was successful (Supplemental Figure 2). The genomic inflation factor $(\lambda \mathrm{GC})[34]$ is 1.05 , suggesting that the population structure of our GWAS is generally acceptable.

When comparing the allele frequencies of the 645,983 SNPs of 70 AOSD cases and 688 population controls, no SNP reached the threshold of genome-wide significance $\left(\mathrm{Pc}<7.8 \times 10^{-8}\right)$. Given AOSD is a rare disease and small sample size used in this study, we set the cut off $P$ value as $1 \times 10^{-6}$ and the top 4 significant SNPs with highly suggestive association are shown in Figure 1 and Table 2. Of these 4 SNPs, two SNPs (rs35910146 and rs6948305) are on the upstream of pseudogenes, and rs9636107 does not match Hardy-Weinberg equilibrium $(P=0.03)$ in the dataset of 688 population controls (Table 2). The SNP rs11102024 on $5^{\prime}$-upstream of CSF1 (colony-stimulating factor 1) gene encoding for M-CSF (macrophage colony-stimulating factor) on chromosome 1p13 showed significant association with AOSD $\left(P=3.70 \times 10^{-7}\right.$, OR (95\% CI): 3.27 (2.07 5.17) (Table 2). By comparison, the association strength between SNPs on HLA regions and AOSD was weaker than that of variants near CSF1 gene (Supplemental Table 1).

3.3. Replication, Meta-Analysis, and Linkage Disequilibrium of Variants near CSF1 Gene. We used the samples of an independent cohort (36 AOSD cases and 200 controls) to replicate the association. Among the 4 SNPs discovered by GWAS, only SNP rs11102024 near CSF1 displayed signifi- cant association with $\operatorname{AOSD}(P=0.022$, OR $(95 \% \mathrm{CI})=2.47$ $(1.20 \sim 5.08)$ ) (Table 3$)$. The meta-analysis of the two datasets from the initial GWAS and replication revealed that the $P$ value of the heterogeneity test between studies $\left(P_{\text {het }}\right)$ was 0.36 , suggesting that there was no difference between both studies ( $P$ value of the heterogeneity test $>0.05)$ (Figure 2, Table 3). Subsequently, we performed combined sample analysis using the genotyping data of 106 AOSD cases and 888 population controls, and the SNP rs11102024 showed strong association with AOSD $\left(P=1.20 \times 10^{-8}\right.$; OR (95\% CI): 3.28 (2.25 4.79)) (Table 3, Figure 2). We used the genetic dataset of East Asian population of the 1,000 genome project for linkage disequilibrium (LD) analysis and identified 11 SNPs having strong LD with rs11102024 $\left(r^{2}>0.7\right)$ (Supplemental Table 2, Supplemental Figure 3). We also sequenced the CSF1 gene; however, there were no missense SNPs with significant association with AOSD (data not shown).

3.4. Increased Plasma M-CSF Levels in AOSD Patients and Correlation with the Activity Score of the Disease. We hypothesized that genetic variant near CSF1 might have an impact on the disease progress/outcome of AOSD. We determined the plasma levels of M-CSF in 82 patients who had available plasma samples. Significantly higher levels of plasma M-CSF were detected in AOSD patients (median $9.31 \mathrm{pg} / \mathrm{mL}$, inter-quartile range (IQR) $6.21-16.91 \mathrm{pg} / \mathrm{mL}$ ) compared with the healthy controls $(n=68)$ (median $5.31 \mathrm{pg} / \mathrm{mL}, \mathrm{IQR}$ $4.12-6.85 \mathrm{pg} / \mathrm{mL}$ ) (nonparametric Mann-Whitney $U$ test, $P<0.0001$ ) (Figure 3(a)). Moreover, significantly higher M-CSF levels were detected in the AOSD patients with activity score $\geq 6 \quad(n=42$; median $10.94 \mathrm{pg} / \mathrm{mL}$; IQR 6.73$20.23 \mathrm{pg} / \mathrm{mL}$ ), compared to those with activity score between 3 and $5 \quad(n=40 ; 7.68 \mathrm{pg} / \mathrm{mL}, \quad \mathrm{IQR} \quad 5.88-11.38 \mathrm{pg} / \mathrm{mL})$ (Figure 3(b)).

3.5. Correlation of Plasma M-CSF Levels, the Genotypes of CSF1, and Disease Outcome of AOSD. The AOSD patients $(n=82)$ were divided into three groups according to the genotype of rs11102024. The highest M-CSF levels were observed in AOSD patients carrying the TT genotype ( $n=10$; median: $20.28 \mathrm{pg} / \mathrm{mL}$; IQR $16.78-33.86 \mathrm{pg} / \mathrm{mL}$ ) compared with the carriers of AA genotype $(n=55$; median: $6.82 \mathrm{pg} / \mathrm{mL}$; IQR $5.88-10.79 \mathrm{pg} / \mathrm{mL}$ ) (nonparametric MannWhitney $U$ test, $P<0.0001)$ and AT genotype $(n=17$; median: $11.61 \mathrm{pg} / \mathrm{mL}$, IQR $8.47-19.98 \mathrm{pg} / \mathrm{mL})(P=0.027)$, respectively (Figure 3(c)). Furthermore, AOSD patients carrying the rs11102024 TT genotype displayed significantly higher M-CSF expressions ( $n=10$; median: $20.28 \mathrm{pg} / \mathrm{mL}$; IQR $16.78-33.86 \mathrm{pg} / \mathrm{mL}$ ) than those with AA/AT genotypes $(n=72 ; 7.95 \mathrm{pg} / \mathrm{mL}$, IQR $6.03-12.73 \mathrm{pg} / \mathrm{mL}) \quad(P<0.0001)$ (Figure 3(d)).

Regarding the disease outcome, 64 of 82 AOSD patients had the systemic pattern, and 18 cases had the chronic articular pattern. Significantly higher M-CSF levels were observed in AOSD patients with the systemic pattern (median $10.79 \mathrm{pg} / \mathrm{mL}$, IQR $6.40-18.53 \mathrm{pg} / \mathrm{mL}$ ) compared to those with the chronic articular pattern $(6.23 \mathrm{pg} / \mathrm{mL}, \mathrm{IQR}$ 4.37-8.41 pg/mL) $(P=0.0007)$ (Figure 3(e)). In addition, the 


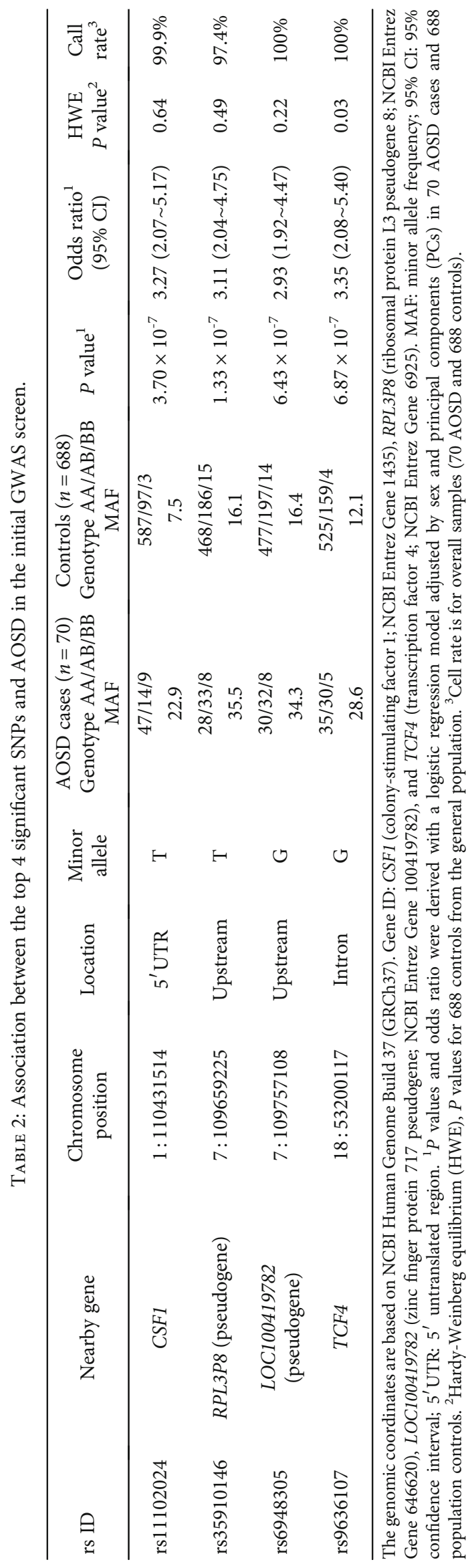




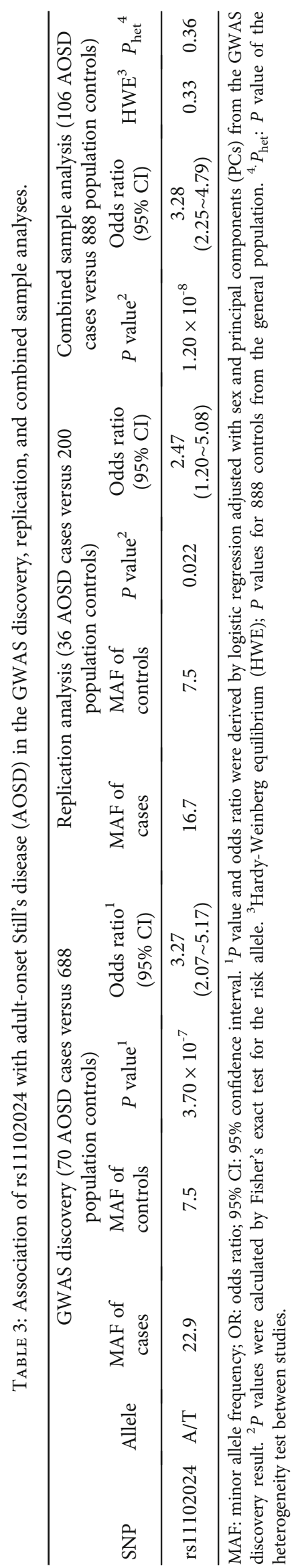




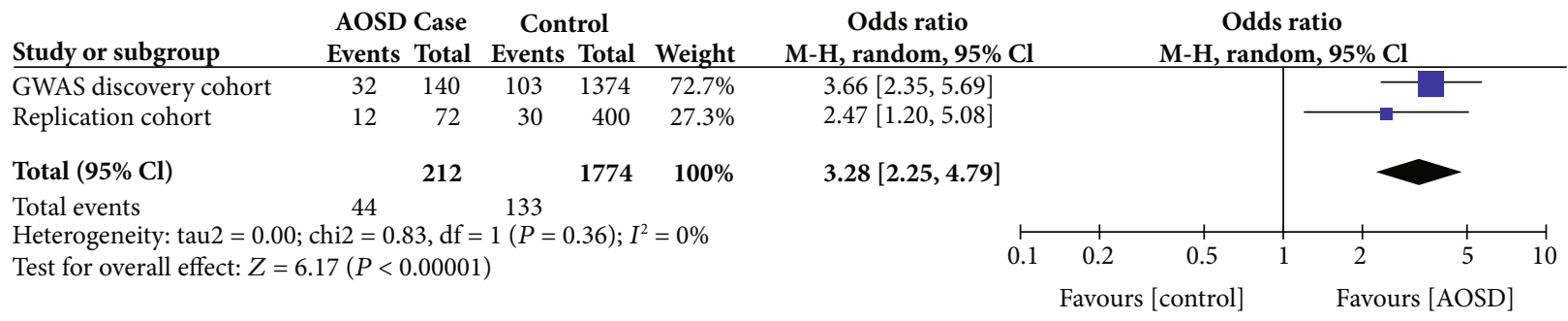

FIGURE 2: Meta-analysis of the genetic association between rs11102024 and adult-onset Still's disease (AOSD). M-H: Mantel-Haenszel test.

AOSD patients with the systemic pattern accounted for $80 \%$ of the rs11102024 TT genotype carriers, comparing to only $20 \%$ with the chronic articular pattern.

\section{Discussion}

AOSD possesses similar clinical presentations with rheumatoid arthritis (RA); however, AOSD does not have RF or significant memorial lymphocyte involvement, and the genetic susceptibility revealed by this study showing no similarity with that of rheumatoid arthritis [35]. Consistent with the previous study, we did not find association between AOSD and $M E F V$ gene mutations [36-38] or other genetic mutations of monogenic autoinflammatory disorders [39, 40]. The systemic juvenile idiopathic arthritis (juvenile-onset Still's disease) has multiple-gene predisposition $[8,41]$, including HLA-DRB1 [21, 42-44]. In addition, both HLA class I and II regions have been reported to be risk loci for AOSD [29]. However, these findings are controversial to the clinical characteristics of juvenile-onset or adult-onset Still's disease, as HLA would implicate the predominant adaptive immune response. In this study, we find that SNPs on HLA class I and class II had only weak association with AOSD, but $5^{\prime}$-upstream variants of CSF 1 reached genomewide significance. The different observations may relate to the enrolling criteria for patients with diverse clinical presentations of Still's disease [18, 44]. Further studies are needed to verify these genetic associations with AOSD in different populations.

CSF1 gene encoding M-CSF has been implicated in the differentiation and activation of monocyte/macrophage lineage $[25,26]$. The increased M-CSF plasma levels have been considered a biomarker for AOSD [22, 25, 26]. In our study, M-CSF expression correlating with AOSD activity and severity is consistent with the results of previous studies $[22,25,26]$. Particularly, our GWAS first demonstrates that SNP rs11102024 on $5^{\prime}$-upstream of CSF1 reached genomewide significance, and the subsequently replication study confirmed the strong genetic association with AOSD. We further identified 11 SNPs having strong LD with rs11102024 $\left(r^{2}>0.7\right)$, and most of them show regulatory potential [45]. As the associated SNP rs 11102024 is only $22 \mathrm{~kb}$ upstream of CSF1 and the nearby LD variants show regulatory potential, we propose that these SNPs may upregulate the expression levels of M-CSF, resulting in the disease of AOSD. In support of the functional roles of associated genetic variants, we indeed found significantly higher levels of plasma M-CSF in our AOSD patients with rs11102024 TT genotype. Taken together, our data suggest that the variants near CSF1 may play important pathogenesis roles to regulate the gene expression and participate in the disease course of AOSD. Further studies are needed to investigate the potential mechanism of action of the genetic variants in the context of AOSD.

The clinical course of AOSD can be divided into two main patterns with different prognoses: systemic pattern (monophasic or polycyclic) and chronic articular pattern. The systemic pattern is characterized by predominantly systemic features including fever, rash, serositis, and organomegaly with or without articular symptomatology. By comparison, the chronic articular pattern is characterized by the severe articular manifestations mimicking rheumatoid arthritis. In this study, we observed higher plasma levels of M-CSF in AOSD patients whose activity score $\geq 6$, cases with systemic pattern, or carriers with rs11102024 TT genotype. These data suggest that rs $11102024 \mathrm{~T}$ allele, which is highly associated with M-CSF plasma levels, could be used to predict the severity and system symptoms in the early phase of AOSD and act as a promising genetic marker for early intervention to improve AOSD outcome.

As AOSD is a polygenetic autoinflammatory disorder, increasing biologic agents are investigated to target its proinflammatory cytokines, such as IL- 1 family (particularly IL- $1 \beta$ and IL-18), IL-6, and TNF- $\alpha$. Growing evidences and clinical trials indicate that anticytokine biologic agents, e.g., anakinra (interleukin-1 receptor antagonist) [46] and tocilizumab (anti-IL-6 receptor antibody) [47], are becoming plausible therapeutic options for the management of AOSD. Recently, Jaguin et al. reported the phenotypic and genomic markers of M-CSF-generated human macrophages polarized towards M1 or M2 subtype upon the action of lipopolysaccharide and interferon- $\gamma$ (for M1) or interleukin- (IL-) 4 (for M2) [48]. The ability of human M-CSF-generated macrophages to polarize toward M1 or M2 subtype was associated with enhanced secretion of TNF- $\alpha$, IL-1 $\beta$, IL-12p40, CXCL10, and IL-10 (for M1) or CCL22 (for M2) [48]. In addition, Bellora et al. reported that M-CSF could induce the expression of membrane-bound IL-18 in human blood monocytes differentiating toward macophages [49]. The enhancement of these macrophage-triggered cytokines was frequently observed in AOSD patients [22, 50]. This functional property of M-CSF highlights the innate immunity and subsequent adaptive immune responses implicated in AOSD pathogenesis. In this study, we report that the novel SNP rs11102024 


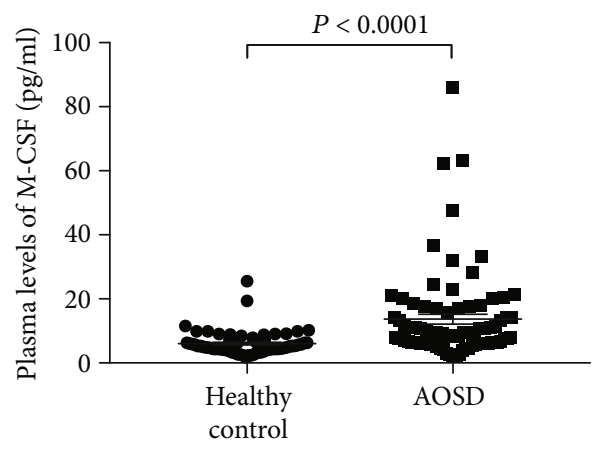

(a)

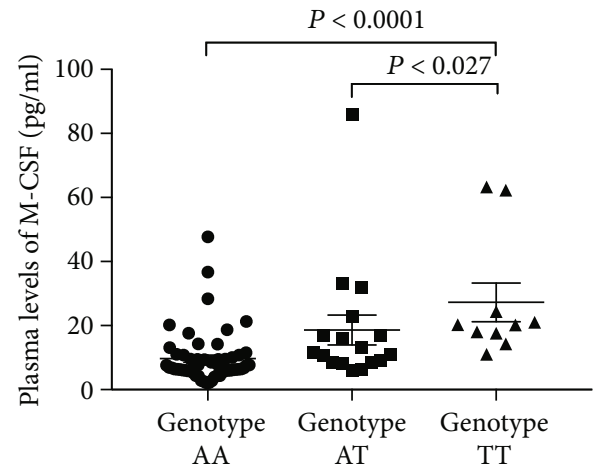

(c)

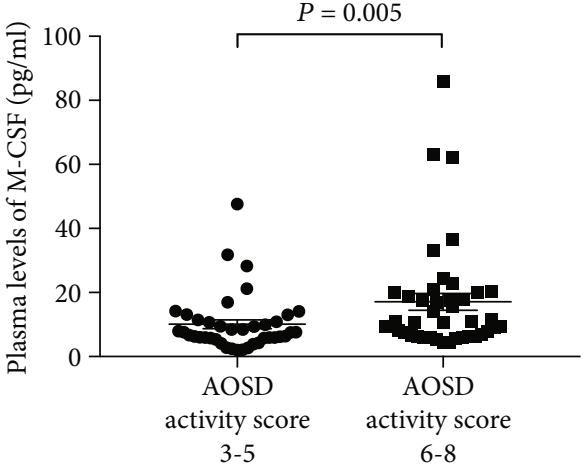

(b)

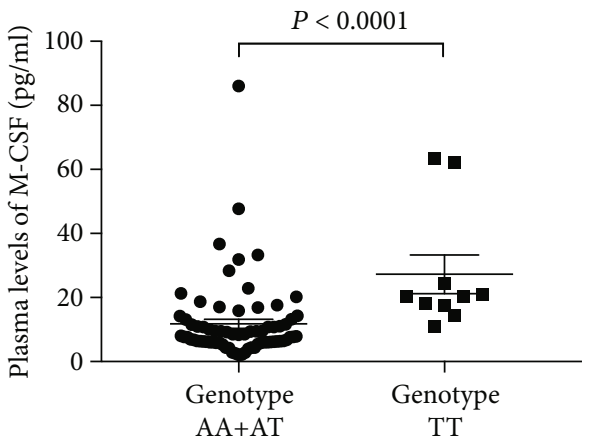

(d)

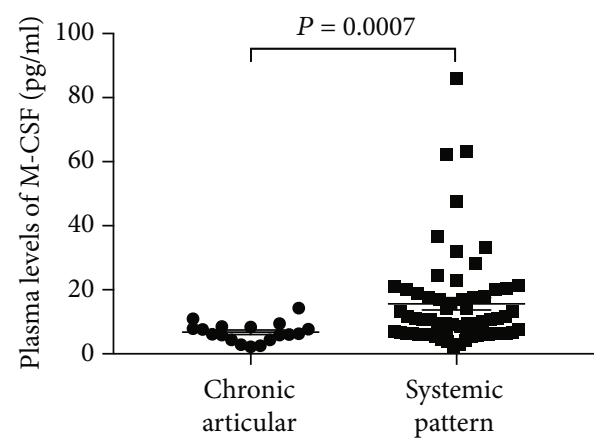

(e)

FIgURE 3: Increase of plasma M-CSF (macrophage colony-stimulating factor) levels in AOSD patients and correlation with the activity score, disease outcomes, and genotypes of SNP rs11102024 nearby CSF1 gene. (a, b) The plasma levels of M-CSF protein were determined in the samples of healthy controls $(n=68)$ and AOSD patients $(n=82)$. (a) A significant increase of plasma M-CSF levels was detected in the AOSD patients compared to those of healthy controls. (b) The plasma levels of M-CSF are significantly increased in AOSD patients with activity scores 6-8, compared to those of AOSD patients with activity scores 3-5. (c, d) The AOSD patients $(n=82)$ were divided into three groups according to the genotype of rs11102024. (c) AOSD patients carrying the SNP rs11102024 TT genotype showed significantly higher levels of M-CSF ( $n=10$, median: $20.28 \mathrm{pg} / \mathrm{mL})$, compared to the carriers of AA genotype $(n=55 ; \mathrm{median}: 6.82 \mathrm{pg} / \mathrm{mL})(P<0.0001)$ and AT genotype $(n=17$; median: $11.61 \mathrm{pg} / \mathrm{mL})(P=0.027)$. (d) A significant increase of plasma M-CSF levels was detected in AOSD patients with rs11102024 TT genotype than those of AA/AT genotypes. (e) Correlation between the plasma levels of M-CSF and disease outcome of 82 AOSD patients (systemic pattern $(n=64)$ and chronic articular pattern $(n=18)$ ). AOSD patients with the systemic pattern showed a significant increase of M-CSF (median: $10.79 \mathrm{pg} / \mathrm{mL}$ ) compared to those with chronic articular pattern (median: $6.23 \mathrm{pg} / \mathrm{mL}$ ). These results are expressed as the mean \pm SEM with each dot representing the data of an individual. Statistical analysis was performed using a two-tailed, nonparametric Mann-Whitney $U$ test.

near CSF1 gene encoding for M-CSF is highly associated with AOSD and links to higher plasma levels of M-CSF, which would be a potential and promising therapeutic target for AOSD.

\section{Conclusion}

Adult-onset Still's disease (AOSD) is a rare, but systemic inflammatory disorder characterized by spiking fever, rash, 
leukocytosis, arthritis, and multisystemic involvement, with increased macrophages and M-CSF. Herein, we identify a novel SNP rs11102024 on the $5^{\prime}$-upstream of CSF1 gene to be significantly associated with AOSD in a GWAS and subsequent replication analysis. Reflecting the function of CSF1, encoding M-CSF which is involved in macrophage differentiation and inflammatory responses, the SNP rs11102024 demonstrated the significant association with plasma M-CSF levels, disease activity, and disease outcome of AOSD. These data suggest that the gene variant rs11102024 nearby CSF1 could be a potential prognostic factor for a disease outcome in AOSD. These results provide a promising initiative to predict and early intervention to improve the treatment and healthcare for AOSD. Further investigation is needed to a better understanding of the pathogenesis of AOSD and clinical implementation for the genetic variants of CSF1.

\section{Abbreviations}

$\begin{array}{ll}\text { AOSD: } & \text { Adult-onset Still's disease } \\ \text { CI: } & \text { Confidence interval } \\ \text { CSF1: } & \text { Colony-stimulating factor } 1 \\ \text { GWAS: } & \text { Genome-wide association study } \\ \text { ELISA: } & \text { Enzyme-linked immunosorbent assay } \\ \text { HWE: } & \text { Hardy-Weinberg equilibrium } \\ \text { LD: } & \text { Linkage disequilibrium } \\ \text { MAF: } & \text { Minor allele frequency } \\ \text { M-CSF: } & \text { Macrophage colony-stimulating factor } \\ \text { OR: } & \text { Odds ratio } \\ \text { PCA: } & \text { Principal component analysis } \\ \text { SNP: } & \text { Single-nucleotide polymorphism } \\ \text { UTR: } & \text { Untranslated region. }\end{array}$

\section{Data Availability}

All data relevant to the study are included in the article or uploaded as supplementary information.

\section{Conflicts of Interest}

The authors declare no conflict of interest.

\section{Authors' Contributions}

All authors made substantive intellectual contributions to the present study and approved the final manuscript. Y-MC and W-TH contributed equally to this work, designed the study, conceived the study, performed clinical assessment as well as data acquisition, and drafted the manuscript. C-WH performed clinical assessments on study subjects, conducted the analysis of data, and drafted the manuscript. W-HC and J-LL designed the study and performed the data analysis. N-RG, W-CC, and Y-SL conceived the study, obtained the laboratory and genomic data, and performed the analysis. $\mathrm{D}-\mathrm{YC}$ and S-I H contributed equally to this work, generated the original hypothesis, designed the study, acquired the clinical data and data analysis, and drafted and revised the manuscript.

\section{Acknowledgments}

This work was supported in part by grants NSC-101-2314B-010-030-MY3, 102-2314-B-010-014-MY3, 105-2628-B010-007-MY3, and 108-2320-B-182A-023-MY3 from the Ministry of Science and Technology, Taiwan; grant TCVGH-YM1030201 from Taichung Veterans General Hospital and National Yang-Ming University; and research grants CIRPG3I0041 and CIRPG3I0021 from Chang Gung Memorial Hospital, Linkou Branch, Taoyuan, Taiwan. We thank the support of members of the Whole-Genome Research Core Laboratory of Human Diseases, Chang Gung Memorial Hospital, Taiwan.

\section{Supplementary Materials}

Supplemental Table 1: association between SNPs on HLA region and AOSD. Supplemental Table 2: proxy variants in linkage disequilibrium (LD) with the SNP 11102024. Supplemental Figure 1: a scatter plot of the principal components analysis (PCA) for the two principal components (PC1 and PC2). Supplemental Figure 2: a quantile-quantile plot of the test statistics in a case-control study for AOSD. Supplemental Figure 3: linkage disequilibrium (LD) analysis of SNP rs11102024 in the East Asian (EAS) population. (Supplementary Materials)

\section{References}

[1] E. G. Bywaters, "Still's disease in the adult," Annals of the Rheumatic Diseases, vol. 30, no. 2, pp. 121-133, 1971.

[2] B. Fautrel, "Adult-onset Still disease," Best Practice \& Research. Clinical Rheumatology, vol. 22, no. 5, pp. 773-792, 2008.

[3] K. J. Evensen and H. C. Nossent, "Epidemiology and outcome of adult-onset Still's disease in Northern Norway," Scandinavian Journal of Rheumatology, vol. 35, no. 1, pp. 48-51, 2006.

[4] Y. F. Asanuma, T. Mimura, H. Tsuboi et al., "Nationwide epidemiological survey of 169 patients with adult still's disease in Japan," Modern Rheumatology, vol. 25, no. 3, pp. 393-400, 2015.

[5] T. Fujii, A. Suwa, T. Mimori, and M. Akizuki, "Chronic arthritis and carpo:metacarpal ratio in Japanese patients with adult Still's disease," The Journal of Rheumatology, vol. 25, no. 12, pp. 2402-2407, 1998.

[6] M. Gerfaud-Valentin, D. Maucort-Boulch, A. Hot et al., "Adult-onset still disease: manifestations, treatment, outcome, and prognostic factors in 57 patients," Medicine, vol. 93, no. 2, pp. 91-99, 2014.

[7] M. Agha-Abbaslou, A. M. Bensaci, O. Dike, M. C. Poznansky, and A. Hyat, "Adult-onset still's disease: still a serious health problem (a case report and literature review)," American Journal of Case Reports, vol. 18, pp. 119-124, 2017.

[8] L. Rossi-Semerano and I. Koné-Paut, "Is Still's Disease an Autoinflammatory Syndrome?," International Journal of Inflammation, vol. 2012, Article ID 480373, 5 pages, 2012.

[9] M. Gerfaud-Valentin, Y. Jamilloux, J. Iwaz, and P. Sève, "Adult-onset Still's disease," Autoimmunity Reviews, vol. 13, no. 7, pp. 708-722, 2014. 
[10] Y. Jamilloux, M. Gerfaud-Valentin, T. Henry, and P. Sève, "Treatment of adult-onset Still's disease: a review," Therapeutics and Clinical Risk Management, vol. 11, pp. 33-43, 2014.

[11] S. Kadavath and P. Efthimiou, "Adult-onset Still's diseasepathogenesis, clinical manifestations, and new treatment options," Annals of Medicine, vol. 47, no. 1, pp. 6-14, 2015.

[12] T. J. Chen, W. H. Chung, C. B. Chen et al., "Methotrexateinduced epidermal necrosis: a case series of 24 patients," Journal of the American Academy of Dermatology, vol. 77, no. 2, pp. 247-255.e2, 2017.

[13] P. Ruscitti and R. Giacomelli, "Pathogenesis of adult onset still's disease: current understanding and new insights," Expert Review of Clinical Immunology, vol. 14, no. 11, pp. 965-976, 2018.

[14] E. Feist, S. Mitrovic, and B. Fautrel, "Mechanisms, biomarkers and targets for adult-onset Still's disease," Nature Reviews Rheumatology, vol. 14, no. 10, pp. 603-618, 2018.

[15] J. M. Wouters, J. van der Veen, L. B. van de Putte, and D. J. de Rooij, “Adult onset Still's disease and viral infections," Annals of the Rheumatic Diseases, vol. 47, no. 9, pp. 764-767, 1988.

[16] M. Kawashima, M. Yamamura, M. Taniai et al., "Levels of interleukin-18 and its binding inhibitors in the blood circulation of patients with adult-onset Still's disease," Arthritis and Rheumatism, vol. 44, no. 3, pp. 550-560, 2001.

[17] T. Sugiura, Y. Kawaguchi, M. Harigai et al., "Association between adult-onset Still's disease and interleukin-18 gene polymorphisms," Genes and Immunity, vol. 3, no. 7, pp. 394399, 2002.

[18] M. L. Miller, S. Aaron, J. Jackson et al., "HLA gene frequencies in children and adults with systemic onset juvenile rheumatoid arthritis," Arthritis and Rheumatism, vol. 28, no. 2, pp. 146150, 1985.

[19] J. M. Wouters, P. Reekers, and L. B. van de Putte, "Adult-onset Still's disease. Disease course and HLA associations," Arthritis and Rheumatism, vol. 29, no. 3, pp. 415-418, 1986.

[20] C. I. Joung, H. S. Lee, S. W. Lee et al., “Association between HLA-DR B1 and clinical features of adult onset Still's disease in Korea," Clinical and Experimental Rheumatology, vol. 21, no. 4, pp. 489-492, 2003.

[21] D. Y. Chen, Y. M. Chen, H. H. Chen, C. W. Hsieh, C. C. Lin, and J. L. Lan, "Functional association of interleukin 18 gene -607 (C/A) promoter polymorphisms with disease course in Chinese patients with adult-onset Still's disease," The Journal of Rheumatology, vol. 36, no. 10, pp. 2284-2289, 2009.

[22] J. H. Choi, C. H. Suh, Y. M. Lee et al., "Serum cytokine profiles in patients with adult onset Still's disease," The Journal of Rheumatology, vol. 30, no. 11, pp. 2422-2427, 2003.

[23] M. Rau, M. Schiller, S. Krienke, P. Heyder, H. Lorenz, and N. Blank, "Clinical manifestations but not cytokine profiles differentiate adult-onset Still's disease and sepsis," The Journal of Rheumatology, vol. 37, no. 11, pp. 2369-2376, 2010.

[24] D. Álvarez-Errico, R. Vento-Tormo, and E. Ballestar, "Genetic and epigenetic determinants in autoinflammatory diseases," Frontiers in Immunology, vol. 8, p. 318, 2017.

[25] K. Matsui, T. Tsuchida, K. Hiroishi et al., "High serum level of macrophage-colony stimulating factor (M-CSF) in adult-onset Still's disease," Rheumatology, vol. 38, no. 5, pp. 477-478, 1999.

[26] J. Maruyama and S. Inokuma, "Cytokine profiles of macrophage activation syndrome associated with rheumatic diseases," The Journal of Rheumatology, vol. 37, no. 5, pp. 967-973, 2010.
[27] T. Asano, H. Furukawa, S. Sato et al., "Effects of HLA-DRB1 alleles on susceptibility and clinical manifestations in Japanese patients with adult onset Still's disease," Arthritis Research \& Therapy, vol. 19, no. 1, p. 199, 2017.

[28] Z. Li, H. L. Liu, J. Chen et al., "BothHLAclass I and II regions identified as genome-wide significant susceptibility loci for adult-onset Still's disease in Chinese individuals," Annals of the Rheumatic Diseases, vol. 79, no. 1, pp. 161-163, 2019.

[29] M. Yamaguchi, A. Ohta, T. Tsunematsu et al., "Preliminary criteria for classification of adult Still's disease," The Journal of Rheumatology, vol. 19, no. 3, pp. 424-430, 1992.

[30] P. Efthimiou, P. K. Paik, and L. Bielory, "Diagnosis and management of adult onset Still's disease," Annals of the Rheumatic Diseases, vol. 65, no. 5, pp. 564-572, 2006.

[31] W. H. Chung, W. C. Chang, Y. S. Lee et al., "Genetic variants associated with phenytoin-related severe cutaneous adverse reactions," JAMA, vol. 312, no. 5, pp. 525-534, 2014.

[32] S. M. Gogarten, T. Bhangale, M. P. Conomos et al., "GWASTools: an R/Bioconductor package for quality control and analysis of genome-wide association studies," Bioinformatics, vol. 28, no. 24, pp. 3329-3331, 2012.

[33] F.-Y. Hu, X. T. Wu, D. M. An, B. Yan, H. Stefan, and D. Zhou, "Phenytoin-induced Stevens-Johnson syndrome with negative HLA-B*1502 allele in mainland China: two cases," Seizure, vol. 20, no. 5, pp. 431-432, 2011.

[34] A. L. Price, N. A. Zaitlen, D. Reich, and N. Patterson, "New approaches to population stratification in genome-wide association studies," Nature Reviews. Genetics, vol. 11, no. 7, pp. 459-463, 2010.

[35] S. Eyre, J. Bowes, D. Diogo et al., "High-density genetic mapping identifies new susceptibility loci for rheumatoid arthritis," Nature Genetics, vol. 44, no. 12, pp. 1336-1340, 2012.

[36] J. J. Kim, J. K. Kim, S. C. Shim et al., "MEFV gene mutations and their clinical significance in Korean patients with adultonset Still's disease," Clinical and Experimental Rheumatology, vol. 31, 3 Suppl 77, pp. 60-63, 2013.

[37] F. Nonaka, K. Migita, Y. Jiuchi et al., "Increased prevalence of MEFV exon 10 variants in Japanese patients with adult-onset Still's disease," Clinical and Experimental Immunology, vol. 179, no. 3, pp. 392-397, 2015.

[38] M. Yashiro, H. Furukawa, T. Asano et al., "Serum amyloid A1 (SAA1) gene polymorphisms in Japanese patients with adultonset Still's disease," Medicine, vol. 97, no. 49, p. e13394, 2018.

[39] G. Giancane, N. M. Ter Haar, N. Wulffraat et al., "Evidencebased recommendations for genetic diagnosis of familial Mediterranean fever," Annals of the Rheumatic Diseases, vol. 74, no. 4, pp. 635-641, 2015.

[40] S. Ozen and Y. Bilginer, "A clinical guide to autoinflammatory diseases: familial Mediterranean fever and next-of-kin," Nature Reviews Rheumatology, vol. 10, no. 3, pp. 135-147, 2014.

[41] D. L. Kastner, I. Aksentijevich, and R. Goldbach-Mansky, "Autoinflammatory disease reloaded: a clinical perspective," Cell, vol. 140, no. 6, pp. 784-790, 2010.

[42] M. Ombrello, E. Remmers, I. Tachmazidou et al., "Genome wide association meta-analysis implicates HLA-DRB1, the BTNL2/HLA-DRA region, and a novel susceptibility locus on chromosome 1 in systemic juvenile idiopathic arthritis," American College of Rheumatology, vol. 65, 2013.

[43] M. J. Ombrello, V. L. Arthur, E. F. Remmers et al., "Genetic architecture distinguishes systemic juvenile idiopathic arthritis 
from other forms of juvenile idiopathic arthritis: clinical and therapeutic implications," Annals of the Rheumatic Diseases, vol. 76, pp. 906-913, 2017.

[44] S. J. Lin, H. C. Chao, and D. C. Yan, "Different articular outcomes of Still's disease in Chinese children and adults," Clinical Rheumatology, vol. 19, no. 2, pp. 127-130, 2000.

[45] N. J. Schork, D. Fallin, and J. S. Lanchbury, "Single nucleotide polymorphisms and the future of genetic epidemiology," Clinical Genetics, vol. 58, no. 4, pp. 250-264, 2000.

[46] S. Castañeda, B. Atienza-Mateo, J. L. Martín-Varillas, J. M. Serra López-Matencio, and M. A. González-Gay, "Anakinra for the treatment of adult-onset Still's disease," Expert Review of Clinical Immunology, vol. 14, no. 12, pp. 979-992, 2018.

[47] S. Castañeda, D. Martínez-Quintanilla, J. L. Martín-Varillas, N. García-Castañeda, B. Atienza-Mateo, and M. A. GonzálezGay, "Tocilizumab for the treatment of adult-onset Still's disease," Expert Opinion on Biological Therapy, vol. 19, no. 4, pp. 273-286, 2019.

[48] M. Jaguin, N. Houlbert, O. Fardel, and V. Lecureur, "Polarization profiles of human M-CSF-generated macrophages and comparison of M1-markers in classically activated macrophages from GM-CSF and M-CSF origin," Cellular Immunology, vol. 281, no. 1, pp. 51-61, 2013.

[49] F. Bellora, R. Castriconi, A. Doni et al., "M-CSF induces the expression of a membrane-bound form of IL-18 in a subset of human monocytes differentiating in vitro toward macrophages," European Journal of Immunology, vol. 42, no. 6, pp. 1618-1626, 2012.

[50] D.-Y. Chen, J. L. Lan, F. J. Lin, and T. Y. Hsieh, "Proinflammatory cytokine profiles in sera and pathological tissues of patients with active untreated adult onset Still's disease," The Journal of Rheumatology, vol. 31, no. 11, pp. 2189-2198, 2004. 